Pacific Journal of Mathematics

ON THE UNIFORMIZATION OF CERTAIN CURVES 


\title{
ON THE UNIFORMIZATION OF CERTAIN CURVES
}

\author{
Peter F. Stiller
}

\begin{abstract}
The uniformization theorem of Poincare and Koebe tells us that every smooth connected algebraic curve $X$ over the complex numbers (or any Riemann surface) has as its universal covering space either the complex projective line $P_{C}^{l}$, the complex numbers $C$, or the complex upper half plane $\mathfrak{S}_{\mathcal{E}}=\{z \in \mathbf{C}$ s.t. $\operatorname{Im} z>0\}$. When the universal covering space is the upper half plane $\mathscr{S}$, we can regard the fundamental group $\pi_{1}(X)$ as a subgroup of $\mathrm{SL}_{2}(\mathbf{R})$ acting as covering transformations via linear fractional transformation. We shall focus on the case $\pi_{1}(X) \subset$ $\mathrm{SL}_{2}(\mathbf{Z})$.
\end{abstract}

Introduction. Uniformization can be described by a class of differential equations on algebraic curves called Fuchsian equations (Poincaré [6] or Griffiths [3]). In this setting the monodromy representation of the differential equation gives us the inclusion of $\pi_{1}(X)$ into $\mathrm{SL}_{2}(\mathbf{R})$ and the solutions can be used to explicitly construct the covering map $\pi: \mathfrak{S} \rightarrow X$ much in the spirit of the Weierstrass $\wp$-function and the most classical case of uniformization, namely the inversion of the elliptic integral. Another classical case occurs when $X=\mathbf{P}_{\mathbf{C}}^{1}-\{0,1, \infty\}$; which leads to the theory of the hypergeometric differential equation.

In this paper we will examine the specific case where $X$ can be uniformized by a subgroup $\Gamma$ of $\mathrm{SL}_{2}(\mathbf{Z})$, that is to say, the case where the map

$$
\pi: \mathfrak{S} \rightarrow \mathfrak{S} / \Gamma \cong X
$$

is the universal covering map. $X$ will thus be a non-compact (because of the presence of cusps) modular curve. We will be particularly interested in giving an explicit description of which curves $X$ arise this way. For example in the case of $\mathbf{P}_{\mathbf{C}}^{1}-\{n$ points $\} n \geq 3$ we will give polynomial equations whose solutions represent those configurations of $n$-points whose complement can be uniformized by $\mathrm{SL}_{2}(\mathbf{Z})$, and the parameters necessary to construct the differential equation. The solutions to the equations will also be explicitly given.

The differential equations involved arise naturally in the theory of elliptic surfaces (Stiller [7]), but for the most part no use will be made of that fact. 
We begin the paper with a discussion of differential equations and how they give rise to uniformazations. We then go on to discuss a specific class of differential equations, called $K$-equations, that arises when the uniformization is given by a subgroup of finite index $\Gamma$ in $\mathrm{SL}_{2}(\mathbf{Z})$. By examining the local behaviour of the solutions of a $K$-equation we are able to determine when it uniformizes. More precisely: Let $X$ be a complete smooth connected curve over $\mathbf{C}$ and $S \subset X$ a finite set of points, then $X-S$ can be uniformized by a $K$-equation $\Lambda_{(q, \lambda)}$ in the sense that the inverse of the multi-valued map

$$
X-S \stackrel{\omega_{1} / \omega_{2}}{\rightarrow} \mathscr{Y}
$$

given by a quotient of solutions $\omega_{1}, \omega_{2}$ of $\Lambda_{(g, \lambda)}$ is the universal cover if and only if the rational function $g$ has poles at each point of $S$ and nowhere else, only triple zeros, and $\mathscr{f}-1$ has only double zeros. It then follows, because of the properties of $K$-equations, that we can find a subgroup $\Gamma$ of finite index in $\mathrm{SL}_{2}(\mathbf{Z})$ with $\pi_{1}(X-S) \cong \Gamma$ such that $X-S \cong \mathfrak{S} / \Gamma$, this last isomorphism being specifically given by the differential equation.

\section{Part I. The Differential Equations}

1. Differential equations. By an algebraic differential equation of second order on a complete smooth connected curve $X$ over $\mathbf{C}$ we shall mean an expression

$$
\Lambda f=\frac{d^{2} f}{d x^{2}}+P \frac{d f}{d x}+Q f=0
$$

where $P, Q, x$ are in the function field of $X$ and $x \notin \mathbf{C}$. The function $x$ will be called a parameter; it will furnish us with a local coordinate at all but a finite set of points. Disregarding these points as well as any where $P, Q$ fail to be regular leaves us with a Zariski-open subset $X_{0}$ of $X$. For every $x \in X_{0}$ the equation has two independent holomorphic solutions $f_{1}, f_{2}$ which form a basis for the space of solutions in a neighborhood of $x$. If we pick $x_{0} \in X_{0}$ as a base point and a basis $f_{1}, f_{2}$ for the space of solutions at $x_{0}$, we can obtain a representation

$$
\pi_{1}\left(X_{0}, x_{0}\right) \stackrel{\rho_{\Lambda}}{\rightarrow} \mathrm{GL}_{2}(\mathbf{C})
$$


by analytic continuation. We will denote by

$$
\left(\begin{array}{l}
f_{1} \\
f_{2}
\end{array}\right) \rightarrow \rho_{\Lambda}(\gamma)\left(\begin{array}{l}
f_{1} \\
f_{2}
\end{array}\right)
$$

the analytic continuation of the basis $f_{1}, f_{2}$ around $\gamma \in \pi_{1}\left(X_{0}, x_{0}\right)$. Note that another choice of basis at $x_{0}$ leads to an equivalent representation. This representation is called the monodromy representation of $\Lambda$.

Observe that on $X_{0}$ the functions $f_{1}, f_{2}$ cannot vanish simultaneously. This can be seen by considering the Wronskian $W$ of the equation (1.1):

$$
W=f_{1} f_{2}^{\prime}-f_{2} f_{1}^{\prime}=e^{-\int P d x}
$$

or simply by solving (1.1) using the method of undetermined coefficients. Now let $\tilde{X}_{0}$ be the universal cover of $X_{0}$, then each choice of basis $f_{1}, f_{2}$ for the space of solutions at $x_{0} \in X_{0}$ gives rise to the map $\omega$ :

$$
\begin{array}{lll}
\tilde{X}_{0} & \stackrel{\omega}{\rightarrow} & \mathbf{P}_{\mathbf{C}}^{1} \\
\pi \downarrow & & \\
X_{0} & &
\end{array}
$$

which is well defined (since $f_{1}, f_{2}$ do not vanish simultaneously on $X_{0}$ ). For different choices of $f_{1}, f_{2}$ the maps differ by a linear fractional transformation of $\mathbf{P}_{\mathbf{C}}^{1}$.

We will be interested in the case where $\omega=f_{1} / f_{2}$ is an analytic isomorphism of $\tilde{X}_{0}$ with $\mathfrak{S}$, the complex upper half plane, and where the monodromy representation $\rho_{\Lambda}$ determined by the analytic continuation of $f_{1}, f_{2}$ consists of matrices in $\mathrm{SL}_{2}(\mathbf{Z})$. First however, we will consider a more general situation by relaxing the assumption that $\omega$ be an isomorphism.

2. $K$-equations. Let $X$ be a complete smooth connected algebraic curve over $\mathbf{C}$ with function field denoted by $K(X)$. After fixing a parameter $x \in K(X)$, consider an algebraic differential equation on $X$.

$$
\Lambda f=\frac{d^{2} f}{d x^{2}}+P \frac{d f}{d x}+Q f=0
$$

with $P$ and $Q$ in $K(X)$ and $f$ are unknown function.

Definition I.2.1. $\Lambda f=0$ is called a $K$-equation if it possesses two solutions, $\omega_{1}$ and $\omega_{2}$, which are holomorphic non-vanishing multivalued functions on some Zariski open subset $X_{0}$ of $X$, satisfying:

(i) $\omega_{1}$ and $\omega_{2}$ form a basis of solutions, 
(ii) for every closed path $\gamma \in \pi_{1}\left(X_{0}\right)$ the analytic continuation of $\left(\begin{array}{c}\omega_{1} \\ \omega_{2}\end{array}\right)$ around $\gamma$ is $M_{\gamma}\left(\begin{array}{c}\omega_{1} \\ \omega_{2}\end{array}\right)$ with $M_{\gamma} \in \mathrm{SL}_{2}(\mathbf{Z})$ (the monodromy representation),

(iii) $\operatorname{Im}\left(\omega_{1} / \omega_{2}\right)>0$ on $X_{0}$ (positivity).

Such a pair of solutions is called a $K$-basis. In addition, since the monodromy is in $\mathrm{SL}_{2}(\mathbf{Z})$, the Wronskian $W=e^{-\int P d x}$ is single-valued. We assume as part of our definition:

(iv) $W \in K(X)$ (This assumption can sometimes be discarded; see remarks following Theorem I.2.6.)

Let $\Lambda f=0$ be a $K$-equation with $K$-basis $\omega_{1}$ and $\omega_{2}$. Consider the function $g=J \circ \omega_{1} / \omega_{2}$,

$$
X_{0} \stackrel{\omega_{1} / \omega_{2}}{\rightarrow} \mathfrak{S} \stackrel{J}{\rightarrow} \mathbf{C}
$$

where $J$ is the elliptic modular function on the upper half plane $\mathfrak{S}$. This $\mathscr{f}$ is a single-valued holomorphic function on $X_{0} \subset X$.

\section{Proposition I.2.2. $g \in K(X)$.}

Proof. This is an application of a result which appears in Kodaira [5] as Theorem 7.3.

Thus to every $K$-equation $\Lambda f=0$ and $K$-basis $\omega_{1}, \omega_{2}$ we associate a rational function $g$ in $K(X)$, which is necessarily non-constant.

Let $X$ be any base curve and $g \in K(X)$ any non-constant rational function on $X$. The problem is to produce a $K$-equation $\Lambda f=0$ with a $K$-basis $\omega_{1}$ and $\omega_{2}$ having $g=J\left(\omega_{1} / \omega_{2}\right)$.

Consider first the $z$-sphere $\mathbf{P}_{\mathbf{C}}^{1}$ and the hypergeometric differential equation

$$
\frac{d^{2} f}{d z^{2}}+\frac{1}{z} \frac{d f}{d z}+\frac{31 / 144 z-1 / 36}{z^{2}(z-1)^{2}} f=0 .
$$

The solution in terms of Riemann's $P$-function is

$$
\begin{aligned}
& P\left\{\begin{array}{ccc}
0 & \infty & 1 \\
-1 / 6 & 0 & 1 / 4 \\
1 / 6 & 0 & 3 / 4
\end{array}\right\} \\
& \quad=z^{-1 / 6}(z-1)^{1 / 4} P\left\{\begin{array}{ccc}
0 & \infty & 1 \\
0 & 1 / 12 & 0 \\
1 / 3 & 1 / 12 & 1 / 2
\end{array}\right\}
\end{aligned}
$$


which is seen to be a hypergeometric function. Thus at $z=0$ we have two solutions

$$
\begin{aligned}
& \eta_{1}=z^{-1 / 6}(z-1)^{1 / 4}{ }_{2} F_{1}\left(\frac{1}{12}, \frac{1}{12} ; \frac{2}{3} ; z\right), \\
& \eta_{2}=z^{1 / 6}(z-1)^{1 / 4}{ }_{2} F_{1}\left(\frac{5}{12}, \frac{5}{12} ; \frac{4}{3} ; z\right)
\end{aligned}
$$

which form a basis. We now let

$$
c=(2-\sqrt{3})\left[\frac{\Gamma(11 / 12)}{\Gamma(7 / 12)}\right]^{2} \frac{\Gamma(2 / 3)}{\Gamma(4 / 3)}
$$

( $\Gamma$ the gamma function), and consider another basis of solutions at $z=0$

$$
\begin{aligned}
& \Phi_{1}=e^{2 \pi i / 3} \eta_{1}+c \eta_{2}, \\
& \Phi_{2}=\eta_{1}-c e^{-\pi i / 3} \eta_{2} .
\end{aligned}
$$

The quotient of these solutions $\Phi(z)=\Phi_{1}(z) / \Phi_{2}(z)$ can be regarded as a multivalued function

$$
\mathbf{P}_{\mathbf{C}}^{1}-\{0,1, \infty\} \stackrel{\Phi}{\rightarrow} \mathbf{P}_{\mathbf{C}}^{1}
$$

However, $\Phi$ is an inverse of the elliptic modular function $J$ (Bateman [1]), that is, $\tau=\Phi(J(\tau))$ for $\tau \in \mathfrak{F}$ the upper half plane. Hence $\Phi$ maps $\mathbf{P}_{\mathbf{C}}^{1}-\{0,1, \infty\}$ to $\mathfrak{S}_{\mathcal{B}}$ and $\Phi_{1}, \Phi_{2}$ form a $K$-basis of solutions for (2.2) which is then a $K$-equation.

We return to the problem posed at the beginning of this section. $X$ will be our base curve, $g \in K(X)$ a non-constant rational function, and $x \in K(X)$ a fixed parameter. We regard $g$ as a map $g: X \rightarrow \mathbf{P}_{\mathbf{C}}^{1}$ onto the sphere. Consider the compositions $\Phi_{1}(f), \Phi_{2}(f)$ as multivalued holomorphic non-vanishing functions on some appropriate Zariski open subset $X_{0}$ of $X$. They satisfy a differential equation easily computed to be:

$$
\begin{aligned}
\frac{d^{2} f}{d x^{2}}+ & \frac{(d q / d x)^{2}-g\left(d^{2} q / d x^{2}\right)}{g(d f / d x)} \frac{d f}{d x} \\
& +\frac{(d q / d x)^{2}((31 / 144) g-(1 / 36))}{g^{2}(g-1)^{2}} f=0 .
\end{aligned}
$$

Clearly

$$
\operatorname{Im} \frac{\Phi_{1}(g)}{\Phi_{2}(q)}>0, \quad J\left(\frac{\Phi_{1}(g)}{\Phi_{2}(q)}\right)=g
$$


and

$$
\left(\begin{array}{l}
\Phi_{1}(q) \\
\Phi_{2}(q)
\end{array}\right) \rightarrow M_{\gamma}\left(\begin{array}{l}
\Phi_{1}(q) \\
\Phi_{2}(q)
\end{array}\right)
$$

$M_{\gamma} \in \mathrm{SL}_{2}(\mathbf{Z})$ under analytic continuation around $\gamma \in \pi_{1}\left(X_{0}\right)$. Note also that

$$
\frac{(d g / d x)^{2}-g\left(d^{2} g / d x^{2}\right)}{g \frac{d f}{d x}}=-\frac{d}{d x} \log \frac{d g / d x}{g}
$$

so the Wronskian

$$
W=\exp \left(-\int\left(-\frac{d}{d x} \log \frac{d g / d x}{g}\right) d x\right)=\frac{d g / d x}{g} \in K(X) .
$$

Therefore (2.4) is a $K$-equation, and:

THEOREM I.2.3. Let $X$ be any base curve and $\mathcal{G} \in K(X)$ non-constant. Then there exists a K-equation $\Lambda f=0$ and a $K$-basis $\omega_{1}, \omega_{2}$ of its solutions such that $J\left(\omega_{1} / \omega_{2}\right)=g$. Those constructed above will be referred to as $S K$-equations, and denoted by $\Lambda=\Lambda_{(q, 1)}$.

COROLlaRY I.2.4. The monodromy representation of any $K$-equation with respect to a fixed $K$-basis of solutions is projectively equivalent (in $\mathrm{PSL}_{2}(\mathbf{Z})$ ) to that of the differential equation (2.4) above with appropriate $\mathscr{G}$.

Proof. It is obvious that $q$ determines the projective monodromy in $\mathrm{PSL}_{2}(\mathbf{Z})$ up to conjugation in $\mathrm{PSL}_{2}(\mathbf{Z})$.

COROLlaRY I.2.5. The monodromy group $\Gamma \subset \mathrm{SL}_{2}(\mathbf{Z})$ of a $K$-equation with respect to a fixed $K$-basis of solutions has finite index in $\mathrm{SL}_{2}(\mathbf{Z})$.

Proōf. Obvious.

We will now determine all $K$-equations. Fix a $K$-equation $\Lambda f=0$ on $X$ with $K$-basis $\omega_{1}, \omega_{2}$ such that $\mathcal{G}=J\left(\omega_{1} / \omega_{2}\right)$. Say

$$
\Lambda f=\frac{d^{2} f}{d x^{2}}+P \frac{d f}{d x}+Q f=0 .
$$

We also consider the $S K$-equation $\tilde{\Lambda}=\tilde{\Lambda}_{(g, 1)}$

$$
\tilde{\Lambda} f=\frac{d^{2} f}{d x^{2}}+\tilde{P} \frac{d f}{d x}+\tilde{Q} f=0
$$


with solutions $\Phi_{1}(q), \Phi_{2}(q)$. Let $X_{0} \subset X$ be a Zariski open set on which both bases $\Phi_{1}(\mathcal{F}), \Phi_{2}(\mathcal{F})$ and $\omega_{1}, \omega_{2}$ are holomorphic non-vanishing multivalued functions. Select a base point $x_{0} \in X_{0}$. By Corollary I.2.4 the monodromy representations in $\mathrm{SL}_{2}(\mathbf{Z})$ of $\Lambda, \tilde{\Lambda}$ with respect to the chosen $K$-bases are projectively equivalent, that is, conjugate in $\operatorname{PSL}_{2}(\mathbf{Z})$. Altering $\omega_{1}, \omega_{2}$ to another $K$-basis if necessary, we can assume the projective representations $\pi_{1}\left(X_{0}, x_{0}\right) \rightarrow \mathrm{PSL}_{2}(\mathbf{Z})$ are equal.

THEOREM I.2.6. There exists an algebraic function $\lambda$ on $X$ with $\lambda^{2} \in$ $K(X)$ such that $\lambda \Phi_{1}(\mathcal{F})=\omega_{1}$ and $\lambda \Phi_{2}(\mathcal{F})=\omega_{2}$, and therefore $\Lambda f=0$ is determined:

$$
\begin{aligned}
& P=\tilde{P}-\frac{d}{d x} \log \lambda^{2} \in K(X), \\
& Q=\tilde{Q}-\tilde{P} \frac{d}{d x} \log \lambda-\frac{d^{2} \lambda / d x^{2}}{\lambda}+2\left(\frac{d \lambda / d x}{\lambda}\right)^{2} \in K(X) .
\end{aligned}
$$

Proof. For every point $x$ near $x_{0}$, the lattices $\tilde{L}_{x}=\mathbf{Z} \Phi_{1}(g(x))+$ $\mathbf{Z} \Phi_{2}(g(x))$ and $L_{x}=\mathbf{Z} \omega_{1}(x)+\mathbf{Z} \omega_{2}(x)$ in $\mathbf{C}$ are homothetic. So locally there is a function $\lambda(x)$ holomorphic non-vanishing such that $\lambda(x) \tilde{L}_{x}=$ $L_{x}$. Suppose near $x_{0}$

$$
\begin{aligned}
& \lambda \Phi_{1}(g)=a \omega_{1}+b \omega_{2}, \\
& \lambda \Phi_{2}(g)=c \omega_{1}+d \omega_{2}
\end{aligned}
$$

with $\left(\begin{array}{ll}a & b \\ c & d\end{array}\right) \in \mathrm{SL}_{2}(\mathbf{Z})$. Now analytically continue around $\gamma \in \pi_{1}\left(X_{0}, x_{0}\right)$. If $\tilde{\lambda}$ denotes the continuation of $\lambda$, we have:

$$
\left(\begin{array}{ll}
\tilde{\lambda} & 0 \\
0 & \tilde{\lambda}
\end{array}\right) M_{\gamma}\left(\begin{array}{l}
\Phi_{1} \\
\Phi_{2}
\end{array}\right)= \pm\left(\begin{array}{ll}
a & b \\
c & d
\end{array}\right) M_{\gamma}\left(\begin{array}{l}
\omega_{1} \\
\omega_{2}
\end{array}\right)
$$

where $\pm M_{\gamma}$ is the mondromy of $\Lambda, \tilde{\Lambda}$ (assumed projectively equal). Therefore

$$
\pm\left(\begin{array}{ll}
a & b \\
c & d
\end{array}\right) M_{\gamma}^{-1}\left(\begin{array}{ll}
a & b \\
c & d
\end{array}\right)^{-1} M_{\gamma}\left(\begin{array}{cc}
\tilde{\lambda} & 0 \\
0 & \tilde{\lambda}
\end{array}\right)\left(\begin{array}{l}
\Phi_{1} \\
\Phi_{2}
\end{array}\right)=\left(\begin{array}{ll}
a & b \\
c & d
\end{array}\right)\left(\begin{array}{l}
\omega_{1} \\
\omega_{2}
\end{array}\right) .
$$

or by (2.8)

$$
\pm\left(\begin{array}{ll}
a & b \\
c & d
\end{array}\right) M_{\gamma}^{-1}\left(\begin{array}{ll}
a & b \\
c & d
\end{array}\right)^{-1} M_{\gamma}\left(\begin{array}{l}
\Phi_{1} \\
\Phi_{2}
\end{array}\right)=\left(\begin{array}{cc}
\lambda / \tilde{\lambda} & 0 \\
0 & \lambda / \tilde{\lambda}
\end{array}\right)\left(\begin{array}{l}
\Phi_{1} \\
\Phi_{2}
\end{array}\right) .
$$

This implies, as

$$
\pm\left(\begin{array}{ll}
a & b \\
c & d
\end{array}\right) M_{\gamma}^{-1}\left(\begin{array}{ll}
a & b \\
c & d
\end{array}\right)^{-1} M_{\gamma} \in \mathrm{SL}_{2}(\mathbf{Z})
$$


that $\lambda / \tilde{\lambda}$ is a complex multiplication of $\tilde{L}_{x}$ for all $x$ near $x_{0}$. But since $q$ is necessarily non-constant, the general point does not admit non-trivial complex multiplication. So $\lambda / \tilde{\lambda}$ is identically \pm 1 , and in fact this requires $\left(\begin{array}{ll}a & b \\ c & d\end{array}\right)= \pm 1$. Therefore $\lambda^{2}$ is single-valued on $X_{0}$.

One can compute that $\omega_{1}=\lambda \Phi_{1}(q)$ and $\omega_{2}=\lambda \Phi_{2}(q)$ then satisfy a differential equation with $P$ and $Q$ as in (2.7). Computing the Wronskian gives $e^{-\int P d x}=e^{-\int \tilde{P} d x} \cdot \lambda^{2}$ up to a constant multiple. However, $e^{-\int P d x}$ is the Wronskian $W$ of $\Lambda$ and $e^{-\int \tilde{P} d x}=(d q / d x) / \mathcal{g}$. Since we assumed $W \in$ $K(X)$, we have $\lambda^{2} \in K(X)$.

REMARK. If we drop the assumption that $W$ is rational, we have then classified all differential equations with the properties of $\mathrm{SL}_{2}(\mathbf{Z})$ monodromy and positivity, $\operatorname{Im}\left(\omega_{1} / \omega_{2}\right)>0$. (The first part of the above proof doesn't depend on $W \in K(X))$.

THEOREM I.2.7. Let $\Lambda f=0$ be a K-equation. Then $\Lambda$ has regular singular points and essentially unipotent local monodromy.

Proof. This may be checked directly from the expression (2.7) for $\Lambda$.

\section{PART II. UNIFORMIZATION}

1. Local properties of $K$-equations and uniformization. Let $X$ be a proper smooth connected curve over $\mathbf{C}$ and let $\Lambda_{(q, \lambda)}$ be a $K$-equation on $X$. On a suitable Zariski open subset $X_{0} \subset X$ the equation $\Lambda_{(g, \lambda)}$ will be holomorphic and will possess two solutions $\omega_{1}, \omega_{2}$ which give a basis for the space of solutions, have $\mathrm{SL}_{2}(\mathbf{Z})$ monodromy, and positivity i.e. $\operatorname{Im}\left(\omega_{1} / \omega_{2}\right)>0$. As in I.§1 this gives rise to a map

$$
\begin{array}{lll}
\tilde{X}_{0} & \stackrel{\omega}{\rightarrow} & \mathfrak{S} \\
\pi \downarrow & & \\
X_{0} & &
\end{array}
$$

where $\tilde{X}_{0}$ is the universal cover of $X_{0}$ and $\omega$ is induced by $\omega_{1} / \omega_{2}$. We shall see in a moment that $\Lambda_{(g, \lambda)}$ has enough singularities so that $\tilde{X}_{0}$ is analytically equivalent to $\mathfrak{S}$. The question we wish to ask is: when is $\omega$ an isomorphism so that $\Lambda_{(g, \lambda)}$ is Fuchsian (Griffiths [3] or Poincare [6]) and uniformizes $X_{0}$. We will then have

$$
\mathfrak{S}_{\mathfrak{E}} \stackrel{\circ \omega^{-1}}{\rightarrow} \mathfrak{S} / \Gamma \cong X_{0}
$$


an explicit description of the uniformization of $X_{0}$ where $\Gamma \subset \mathrm{SL}_{2}(\mathbf{Z})$ is the mondromy group for $\omega_{1}, \omega_{2}$.

At this point, we need to calculate the local behavior of $\Lambda_{(g, \lambda)}$ at a point in $X-X_{0}$ in order to determine when an equation of this type could uniformize. By Theorem I.2.6

$$
\Lambda_{(g, \lambda)} f=\frac{d^{2} f}{d x^{2}}+P \frac{d f}{d x}+Q f=0
$$

where

$$
\begin{aligned}
P= & \frac{(d g / d x)^{2}-g\left(d^{2} g / d x^{2}\right)}{g(d g / d x)}-\frac{d}{d x} \log \lambda^{2}, \\
Q= & \frac{(d g / d x)^{2}((31 / 144) g-(1 / 36))}{g^{2}(g-1)^{2}} \\
& -\left[\frac{(d g / d x)^{2}-g\left(d^{2} g / d x^{2}\right)}{g(d q / d x)}\right] \frac{d}{d x} \log \lambda \\
& -\frac{d^{2} \lambda / d x^{2}}{\lambda}+2\left(\frac{d \lambda / d x}{\lambda}\right)^{2} .
\end{aligned}
$$

Now the "parameter" $x$ does not provide a good local parameter everywhere and could therefore introduce singularities. This however is not the case; if $t$ is a local parameter at any point in $X$ then when we express $\Lambda_{(g, \lambda)}$ locally it retains the above form with $t$ in place of $x$, i.e.

$$
\begin{aligned}
& P=\frac{(d f / d t)^{2}-g\left(d^{2} g / d t^{2}\right)}{g(d f / d t)}-\frac{d}{d t} \log \lambda^{2}, \\
& Q=\text { etc. }
\end{aligned}
$$

We see that the global "parameter" $x$ plays no role in determining the singularities. Thus the above form can be used in local calculations.

We will begin by calculating the local behaviour of an $S K$-equation $\Lambda_{(g, 1)}($ i.e. assume $\lambda=1)$ :

Case 1. $q$ has a pole of order $n \geq 1$. In terms of a local coordinate $t$, $g=c_{-n} / t^{n}+\cdots$. An easy calculation gives

$$
\begin{aligned}
& P=\frac{1}{t}+\text { holomorphic, } \\
& Q=\frac{0}{t^{2}}+\frac{q-1}{t}+\text { holomorphic. }
\end{aligned}
$$


Since our equation has regular singular points (as we shall see in the course of these calculations) we can apply well known techniques to determine solutions (see Ince [4], Griffiths [3], or Deligne [2]). The indicial equation is

$$
I(v)=v^{2}+\left(p_{-1}-1\right) v+q_{-2}
$$

where $P=p_{-1} / t+\cdots$ and $Q=q_{-2} / t^{2}+\cdots$. In this case

$$
I(v)=v^{2}
$$

and the exponents are 0,0 . Thus the equation has a basis of solutions of the form

$$
\begin{aligned}
& u_{2}=1+\cdots, \\
& u_{1}=u_{2}\left(\frac{1}{2 \pi i} \log t+\text { holomorphic }\right)
\end{aligned}
$$

so that the local monodromy is $\left(\begin{array}{ll}1 & 1 \\ 0 & 1\end{array}\right)$. To reintroduce $\lambda$ is simple. $\Lambda_{(g, \lambda)}$ will have solutions $\lambda u_{1}, \lambda u_{2}$. So as $\lambda^{2} \in K(X)$, if $\lambda^{2}=t^{r}$. (holomorphic nonvanishing) then the new exponents are $r / 2, r / 2$ and the local monodromy is $\pm\left(\begin{array}{ll}1 & 1 \\ 0 & 1\end{array}\right)$. Note that $u_{1}, u_{2}$ need not be a $K$-basis. Using Kodaira's classification for the monodromy at the singular fibers of an elliptic surface one can show that if $\omega_{1}, \omega_{2}$ are a $K$-basis then after "shifting the cusp to $\infty$ " we have that the continuation of $\omega_{1}, \omega_{2}$ around the singularity gives $\pm\left(\begin{array}{ll}1 & n \\ 0 & 1\end{array}\right)$ where $n>0$ is the order of the pole of $q$.

Case 2. $g$ has a zero of order $n \geq 1$. Then

$$
\begin{aligned}
& P=\frac{1}{t}+\text { holomorphic, } \\
& Q=\frac{-n^{2} / 36}{t^{2}}+\cdots
\end{aligned}
$$

The indicial equation is

$$
I(v)=v^{2}-\frac{n^{2}}{36}
$$

so the exponents are $\pm n / 6$. If $n \neq 0 \bmod 3$ then the exponents differ by $n / 3 \notin \mathbf{Z}$, so there exist two solutions

$$
\begin{aligned}
& u_{1}=t^{-n / 6}(1+\text { higher order in } t), \\
& u_{2}=t^{n / 6}(1+\text { higher order in } t) .
\end{aligned}
$$

If $n \equiv 0 \bmod 3$ one must check certain higher order conditions to insure that no logarithmic behaviour occurs. One may also appeal again to the 
theory of elliptic surfaces where, since $q=0$, the monodromy must be of finite order. Thus

$$
\begin{aligned}
& u_{1}=t^{-n / 6}(1+\text { higher order in } t), \\
& u_{2}=t^{n / 6}(1+\text { higher order in } t)
\end{aligned}
$$

still gives a basis for the space of solutions and the monodromy is $\pm\left(\begin{array}{ll}1 & 0 \\ 0 & 1\end{array}\right)$.

Case 3. $g=1$ to order $n>0$. Then

$$
\begin{aligned}
& P=\frac{-n+1}{t}+\text { holomorphic, } \\
& Q=\frac{(3 / 16) n^{2}}{t^{2}}+\cdots
\end{aligned}
$$

The indicial equation is

$$
I(v)=v^{2}-n v+\frac{3}{16} n^{2}
$$

so the exponents are $n / 4$ and $3 n / 4$. If $n$ is odd then the exponents differ by $n / 2 \notin \mathbf{Z}$ so there is a basis of solutions of the form

$$
\begin{aligned}
& u_{1}=t^{n / 2}(1+\text { higher order terms in } t), \\
& u_{2}=t^{3 n / 2}(1+\text { higher order terms in } t) .
\end{aligned}
$$

As before when $n$ is even we must check certain higher order conditions in order to show that there is no logarithmic behaviour and this can be done. Thus the above form is valid even when $n$ is even.

Case 4. $q \neq 0,1, \infty$ but ord $d q=n>0$. Then

$$
\begin{aligned}
& P=\frac{-n}{t}+\cdots, \\
& Q=\text { holomorphic. }
\end{aligned}
$$

So the indicial equation $I(v)=v^{2}+(-n-1) v$ and the exponents are $(0, n+1)$. Since these differ by an integer, higher order conditions must again be checked. However all is well, and there is a basis of solutions of the form

$$
\begin{aligned}
& u_{1}=1+\text { higher order terms in } t, \\
& u_{2}=t^{n+1}(1+\text { higher order terms in } t) .
\end{aligned}
$$

Note that at such points any $K$-basis $\omega_{1}, \omega_{2}$ of $\Lambda$ must extend with $\omega_{1}, \omega_{2}$ holomorphic non-vanishing. This follows because $\omega_{1} / \omega_{2}\left(\right.$ or $\left.\omega_{2} / \omega_{1}\right)$ is 
holomorphic at $t=0$ with positive (or negative) imaginary part at all nearby points. However

$$
\frac{d}{d t}\left(\omega_{1} / \omega_{2}\right) \text { is } \frac{-W}{\omega_{2}^{2}}
$$

where $W$ is the Wronskian of $\Lambda$ which is $(d q / d t) / g$ or more generally $\lambda^{2}((d q / d t) / f)$. Thus $d / d t\left(\omega_{1} / \omega_{2}\right)$ vanishes at these points and the map from a neighborhood of $t=0$ to $\mathfrak{S}$ given by $\omega_{1} / \omega_{2}$ is ramified.

Case 5: At all remaining points $\Lambda_{(g, 1)}$ is holomorphic.

Finally, to pass from $\Lambda_{(g, 1)}$ to a description of the singularities of $\Lambda_{(g, \lambda)}$ is very simple and is carried out as outlined in Case 1.

THEOREM II.1.1. A K-equation $\Lambda_{(q, \lambda)}$ on a complete smooth connected curve over $\mathbf{C}$ will uniformize a Zariski open subset $X_{0} \subset X$ if and only if the function $q$ has a pole at each point in $X-X_{0}$ with only third order zeros, second order ones, and ord $d q \neq 0$ elsewhere.

Proof. We can reduce immediately to the case $\lambda=1$ and consider the $S K$-equation $\Lambda_{(q, 1)}$. This is because the multivalued inverse of the universal covering map is given by a quotient of solutions $\omega_{1} / \omega_{2}$ and $\lambda \omega_{1} / \lambda \omega_{2}$ $=\omega_{1} / \omega_{2}$.

Now if $X_{0} \subset X$ is to be uniformized by $\Lambda_{(g, 1)}$ we must have the universal covering map represented as

$$
\mathfrak{S}_{\mathfrak{E}} \stackrel{\text { " } \stackrel{-1}{\rightarrow}}{\rightarrow} \mathfrak{\mathcal { E }} / \Gamma \cong X_{0}
$$

where $\omega=\omega_{1} / \omega_{2}$ and $\Gamma$ is the mondromy group of $\Lambda_{(g, 1)}$ which is of finite index in $\mathrm{SL}_{2}(\mathbf{Z})$. This means that the missing points in $X-X_{0}$ must correspond to the cusps of $\Gamma \subset \mathrm{SL}_{2}(\mathbf{Z})$. The inverse to the covering map at such a missing point (which is the map given by $\omega=\omega_{1} / \omega_{2}$ ) must therefore exhibit logarithmic behaviour. Our calculations above show that this occurs only in Case 1, i.e. when $g$ has a pole. So the support of the poles of $g$ must be the set of missing points $X-X_{0}$.

Since there can be no algebraic singularity present in $X_{0}$ the equation $\Lambda_{(g, 1)}$ must have exponents differing by an integer at every other singularity of $\Lambda_{(q, 1)}$. Thus $q$ must have zeros each of order $\equiv 0 \bmod 3$ and ones each of order $\equiv 0 \bmod 2$.

Now $\omega_{1} / \omega_{2}$ must extend to a multivalued holomorphic function on $X_{0}$. (This was discussed in Case 4 and the same argument holds in Cases 2 
and 3; namely $\omega_{1} / \omega_{2}$ will be single-valued since the exponents differ by an integer and $\operatorname{Im} \omega_{1} / \omega_{2}>0$ at all nearby values.)

Thus we have a well-defined map $\omega=\omega_{1} / \omega_{2}$,

$$
\begin{array}{ll}
\tilde{X}_{0} & \stackrel{\omega}{\rightarrow} \\
\pi & \mathscr{S} \text { the upper-half-plane } \\
X_{0} &
\end{array}
$$

where $\tilde{X}_{0}$ is the universal cover. The map is clearly onto by the construction of the equation $\Lambda_{(g, 1)}$, see I.\$2. Moreover it will be unramified if and only if on $X_{0}$, all zeros are third order, all ones are second order, and ord $d q=0$ elsewhere.

Thus if $\Lambda_{(q, 1)}$ uniformizes $X_{0} \subset X$ it must have the properties listed.

The converse holds because given any $\Lambda_{(g, 1)}$ with the listed properties we can let $X_{0}$ be the complement of the poles of $q$ and produce a map $\omega$ from $\tilde{X}_{0}=\mathfrak{S}$ to $\mathfrak{S}$ which is unramified. It must therefore be an isomorphism.

Note. To see that $\tilde{X}_{0} \cong \mathfrak{S}$ we need only see that when $X=\mathbf{P}_{\mathbf{C}}^{1}$ and $\mathscr{g}$ has the above properties that $\mathcal{G}$ has more than 2 distinct poles. Consider the map

$$
X=\mathbf{P}_{\mathbf{C}}^{1} \stackrel{g}{\rightarrow} \mathbf{P}_{\mathbf{C}}^{1}
$$

and apply the Hurwitz Theorem. $q$ must have $6 k$ poles $k \geq 1$ (possibly multiple), $2 k$ zeros each of order 3 and $3 k$ ones of order 2 and no other ramification. Thus

$$
2\left(\text { genus } \mathbf{P}_{\mathbf{C}}^{1}\right)-2=(\operatorname{deg} \mathcal{g})\left(2\left(\text { genus } \mathbf{P}_{\mathbf{C}}^{1}\right)-2\right)+\sum \text { ramification }
$$

gives

$$
-2=6 k(-2)+7 k+(6 k-\# \text { poles })
$$

or

$$
k+2=\# \text { poles. }
$$

Thus \#poles is $\geq 3$.

2. Uniformization of $\mathbf{P}_{\mathbf{C}}^{\mathbf{1}}$-\{points $\}$ and remarks on the general case. Consider the complex projective line $\mathbf{P}_{\mathbf{C}}^{1}$ and a finite set of $n$ points $S=\left\{s_{1}, \ldots, s_{n}\right\} \subset \mathbf{P}_{\mathbf{C}}^{1}$ with $n \geq 3$. 
Question II.2.1. Is there a subgroup $\Gamma \subset \mathrm{SL}_{2}(\mathbf{Z})$ of finite index so that $\mathfrak{S} / \Gamma \cong \mathbf{P}_{\mathbf{C}}^{1}-S$ and

$$
\mathfrak{S} \rightarrow \mathfrak{S} / \Gamma \cong \mathbf{P}_{\mathbf{C}}^{1}-S
$$

exhibits $\mathfrak{\mathcal { E }}$ as the universal cover.

PROPOSITION. II.2.2. T has no elliptic points.

Proposition. II.2.3. $\Gamma / \Gamma \cap \pm\left(\begin{array}{ll}1 & 0 \\ 0 & 1\end{array}\right)=\bar{\Gamma}$ has index $\mu=6(n-2)$ in $\operatorname{PSL}_{2}(\mathbf{Z})$.

Proof. The genus formula for $\mathfrak{\mathcal { E }} / \bar{\Gamma}$ is

$$
g=1+\frac{\mu}{12}-\frac{v_{2}}{4}-\frac{v_{3}}{3}-\frac{v_{\infty}}{2}
$$

where $g$ is the genus, $\mu$ is the index of $\bar{\Gamma}$ in $\operatorname{PSL}_{2}(\mathbf{Z})$ as above, $v_{2}, v_{3}$ are the number of elliptic points of order 2 and 3 respectively, and $v_{\infty}$ is the number of cusps. We get

$$
0=1+\frac{\mu}{12}-\frac{n}{2}
$$

or

$$
\mu=6(n-2) \text {. }
$$

We now choose a coordinate $z$ on $\mathbf{P}_{\mathbf{C}}^{1}$ so that $\infty \notin S$. If $\Gamma$ exists then there exists a Fuchsian differential equation giving the uniformization. It must be a second order algebraic differential equation, with regular singular points, $\mathrm{SL}_{2}(\mathbf{Z})$-monodromy, and positivity. But we have classified these - namely the $K$-equations. Thus

THEOREM II.2.4. $\Gamma$ exists if and only if $\mathbf{P}_{\mathbf{C}}^{1}-S$ can be uniformized by $a$ K-equation.

We can actually find an $S K$-equation $\Lambda_{(q, 1)}$ which works as we only care about the quotient of solutions. $\mathscr{q}$ must be a rational function on $\mathbf{P}_{\mathbf{C}}^{1}$ with a pole (possibly multiple) at each point of $S$ with third order zeros, second order ones, and ord $d q=0$ elsewhere (including $\infty$ ).

Write valence $g=6 k, k \geq 1$ (valence $=\#$ poles $=\#$ zeros $=\#$ ones so must be $\equiv 0 \bmod 6)$. Let $a_{1}, \ldots, a_{2 k}$ be its zeros and $b_{1}, \ldots, b_{3 k}$ its ones.

Proposition II.2.5. $n=k+2$. 
Proof. The zeros and ones of $q$ account for $7 k$ zeros of $d q$. Thus $d q$ must have $7 k+2$ poles at the points $s_{1}, \ldots, s_{n}$ counted with multiplicitly. Say the order is $e_{i} \geq 1$ at $s_{i}, i=1, \ldots, n$. Then

$$
\sum_{i=1}^{n} e_{i}=\text { valence } g=6 k
$$

and

$$
\sum_{i=1}^{n}\left(e_{i}+1\right)=7 k+2
$$

Thus $n=k+2$.

EXAmple. $k=1, n=3$. This is the classical hypergeometric case $\mathbf{P}_{\mathbf{C}}^{1}-3$ points.

Now

$$
\frac{g}{g-1}=c \frac{\left(z-a_{1}\right)^{3} \cdots\left(z-a_{2 k}\right)^{3}}{\left(z-b_{1}\right)^{2} \cdots\left(z-b_{3 k}\right)^{2}}, \quad c \in \mathbf{C}-\{0,1\}
$$

(if $c=1, q$ has a pole at $\infty$ but $\infty \notin S$ ). This function takes value one at each missing point $s_{1}, \ldots, s_{n}$ with multiplicity $e_{1} \cdots e_{n}$ as above. Thus we get the relation:

$$
\text { (2.1) } \begin{aligned}
& \prod_{i=1}^{n}\left(z-s_{l}\right)^{e_{i}} \\
& \quad=\frac{1}{1-c}\left[\left(z-b_{1}\right)^{2} \cdots\left(z-b_{3 k}\right)^{2}-c\left(z-a_{1}\right)^{3} \cdots\left(z-a_{2 k}\right)^{3}\right]
\end{aligned}
$$

which must hold as polynomials in $z$.

This gives $6 k$ equations in the $5 k+1$ unknowns $c$, $a_{1}, \ldots, a_{2 k}, b_{1}, \ldots, b_{3 k}$ which if solvable provides $q$ and allows us to answer our original question II.2.1. in the affirmative, i.e. there will exists $\Gamma \subset$ $\mathrm{SL}_{2}(\mathbf{Z})$ of finite index such that $\mathscr{S} / \Gamma \cong \mathbf{P}_{\mathbf{C}}^{1}-S$ and exhibits $\mathscr{S}$ as the universal cover.

Of course we get a set of equations for each choice in assigning the exponents $e_{i}$, but there are only finitely many choices. Note that freeing up the $s_{l}$ leads to a system of $6 k$ equations in $6 k+3$ unknowns but as three points may always be prespecified in $\mathbf{P}_{\mathbf{C}}^{1}$ this is really $6 k$ equations in $6 k$ unknowns.

In the general case, we still have valence $g=6 k$. Using the same notation, i.e., $s_{1} \cdots s_{n} \in X$ the poles of $\mathcal{G}, e_{1}, \ldots, e_{n}$ their respective orders, 
etc. we have

Proposition II.2.6. $n=k-2 g+2$.

Proof. As before $a_{1}, \ldots, a_{2 k}, b_{1}, \ldots, b_{3 k}$ contribute $7 k$ zeros to $d q$. Thus we must account for $7 k-2 g+2$ poles. That means

$$
\sum_{i=1}^{n} e_{i}+1=7 k-2 g+2
$$

but

$$
\sum_{i=1}^{n} e_{i}=6 k
$$

and the result follows.

Since $n \geq 1$ we must have valence $g \geq 12 g-6$, i.e. $k \geq 2 g-1$.

The difficulty in higher genus comes in trying to construct $g$.

\section{REFERENCES}

1. Bateman Manuscript Project, Higher Transcendental Functions, McGraw-Hill Co., New York (1953).

2. P. Deligne, Equations Différentieles à Singuliers Réguliers, Lecture Notes in Math., 163, Springer-Verlag, Berlin-Heidelberg-New York (1970).

3. P. Griffiths, Differential Equations on Algebraic Varieties, Princeton Lectures, unpublished.

4. E. Ince, Ordinary Differential Equations, Dover Publications, New York (1956).

5. K. Kodaira, On compact analytic surfaces II, Ann. Math., 77 (1963), 563-626.

6. H. Poincaré, Sur les Groupes des Équations Linéaires, Oeuvres, Tome II, Gauthier-Villars, Paris, 1916, 300-401.

7. P. Stiller, Differential equations associated with elliptic surfaces, to appear J. Math. Soc. Japan.

Received January 29, 1981 and in revised form January 11, 1982. Support for this research was provided by a NATO Postdoctral Fellowship and the National Science Foundation.

TEXAS A \& M UNIVERSTTY

College Station, TX 77843 


\section{PACIFIC JOURNAL OF MATHEMATICS}

\section{EDITORS}

Donald BabbitT (Managing Editor)

University of California

Los Angeles, CA 90024

Hugo Rossi

University of Utah

Salt Lake City, UT 84112

C. C. Moore and Arthur Ogus

University of California

Berkeley, CA 94720
J. DugunduI

Department of Mathematics

University of Southern California

Los Angeles, CA 90089-1113

R. FinN and H. SAmelson

Stanford University

Stanford, CA 94305

\section{ASSOCIATE EDITORS}
R. ARENS
E. F. BECKENBACH
B. H. NEUMANN
F. WOLF
K. YosHIDA (1906-1982)

\section{SUPPORTING INSTITUTIONS}

UNIVERSITY OF ARIZONA

UNIVERSITY OF BRITISH COLUMBIA

CALIFORNIA INSTITUTE OF TECHNOLOGY

UNIVERSITY OF CALIFORNIA

MONTANA STATE UNIVERSITY

UNIVERSITY OF NEVADA, RENO

NEW MEXICO STATE UNIVERSITY

OREGON STATE UNIVERSITY
UNIVERSITY OF OREGON

UNIVERSITY OF SOUTHERN CALIFORNIA

STANFORD UNIVERSITY

UNIVERSITY OF HAWAII

UNIVERSITY OF TOKYO

UNIVERSITY OF UTAH

WASHINGTON STATE UNIVERSITY

UNIVERSITY OF WASHINGTON 


\section{Pacific Journal of Mathematics}

Vol. 107, No. $1 \quad$ January, 1983

John Kelly Beem and Phillip E. Parker, Klein-Gordon solvability and the

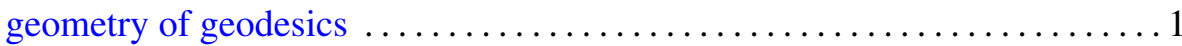

David Borwein and Amnon Jakimovski, Transformations of certain

sequences of random variables by generalized Hausdorff matrices ..... 15

Willy Brandal and Erol Barbut, Localizations of torsion theories . . . . . . . 227

John David Brillhart, Paul Erdős and Richard Patrick Morton, On sums

of Rudin-Shapiro coefficients. II ........................... 39

Martin Lloyd Brown, A note on tamely ramified extensions of rings $\ldots \ldots \ldots 71$

Chang P'ao Ch'ên, A generalization of the Gleason-Kahane-Żelazko

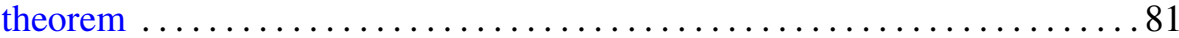

I. P. de Guzman, Annihilator alternative algebras $\ldots \ldots \ldots \ldots \ldots \ldots$. . . . 89

Ralph Jay De Laubenfels, Extensions of $d / d x$ that generate uniformly

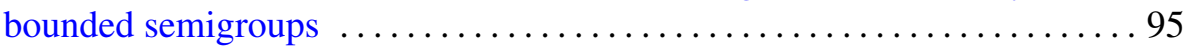

Patrick Ronald Halpin, Some Poincaré series related to identities of $2 \times 2$

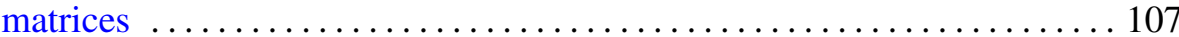

Fumio Hiai, Masanori Ohya and Makoto Tsukada, Sufficiency and

relative entropy in $*$-algebras with applications in quantum systems . . . 117

Dean Robert Hickerson, Splittings of finite groups $\ldots \ldots \ldots \ldots \ldots \ldots \ldots 14$

Jon Lee Johnson, Integral closure and generalized transforms in graded

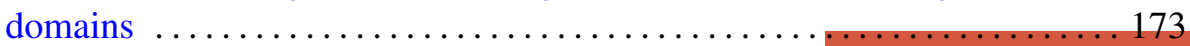

Maria Grazia Marinari, Francesco Odetti and Mario Raimondo, Affine

curves over an algebraically nonclosed field . ................. 179

Douglas Shelby Meadows, Explicit PL self-knottings and the structure of

PL homotopy complex projective spaces $\ldots \ldots \ldots \ldots \ldots \ldots \ldots \ldots \ldots$

Charles Kimbrough Megibben, III, Crawley's problem on the unique

$\omega$-elongation of $p$-groups is undecidable .................... 205

Mary Elizabeth Schaps, Versal determinantal deformations $\ldots \ldots \ldots \ldots 213$

Stephen Scheinberg, Gauthier's localization theorem on meromorphic

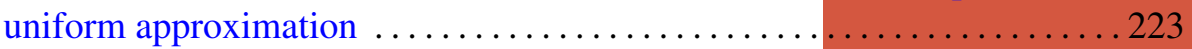

Peter Frederick Stiller, On the uniformization of certain curves . . . . . . . 229

Ernest Lester Stitzinger, Engel's theorem for a class of algebras . . . . . . . . 245

Emery Thomas, On the zeta function for function fields over $F_{p} \ldots \ldots \ldots 251$ 